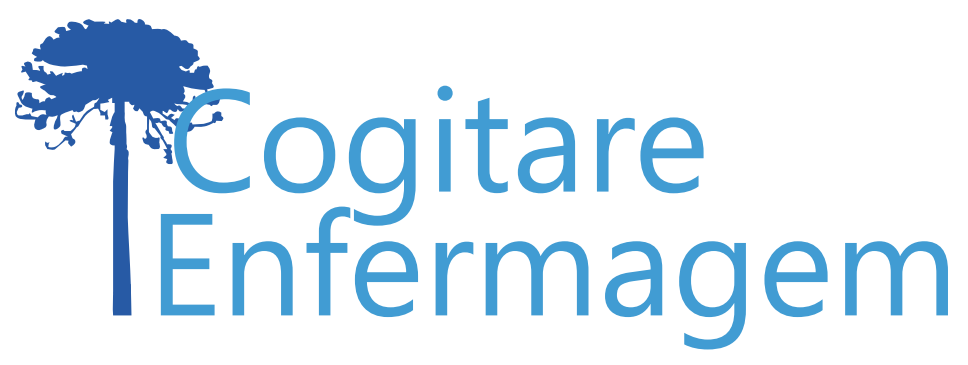

\title{
ADESÃO AO TRATAMENTO NO TRANSPLANTE DE FÍGADO: REVISÃO INTEGRATIVA
}

Priscilla Caroliny de Oliveira开, Felippe Pires Deta², Heloísa Barboza Paglione ${ }^{3}$, Samantha Mucci ${ }^{4}$, Janine Schirmer ${ }^{5}$, Bartira de Aguiar Roza ${ }^{6}$

\section{RESUMO}

Objetivo: avaliar as intervenções da equipe multiprofissional que proporcionam a melhora da adesão ao tratamento após o transplante de fígado.

Método: revisão integrativa da literatura. Os critérios de inclusão foram artigos publicados entre 2012 e 2017, nos idiomas português, inglês e espanhol, realizados na população adulta submetida ao transplante hepático.

Resultado: a busca inicial resultou em 84 publicações sendo selecionados 10 estudos. Os dados foram tabulados de acordo com a relevância do conteúdo, objetivos, tipo de intervenção, número de pacientes e nível de evidência. Foram encontradas quatro categorias analíticas: intervenções educativas; adoção de um plano terapêutico individual; alteração do regime imunossupressor; suporte emocional, psicológico e fortalecimento da rede de apoio.

Conclusão: a equipe multiprofissional deve prover informações sobre o processo de transplantação, realizar o manejo adequado da imunossupressão, avaliar regularmente o paciente quanto aos sinais de depressão, ansiedade, crenças e valores, e instituir um plano de cuidados individualizado. DESCRITORES: Transplante de fígado; Cooperação do paciente; Adesão à medicação; Equipe de assistência ao paciente; Revisão.

COMO REFERENCIAR ESTE ARTIGO:

Oliveira PC de, Deta F, Paglione HB, Mucci S, Schirmer J, Roza B de A. Adesão ao tratamento no transplante de fígado: revisão integrativa. Cogitare enferm. [Internet]. 2019 [acesso em "colocar data de acesso, dia, mês abreviado e ano"]; 24. Disponível em: http://dx.doi.org/10.5380/ce.v24i0.58326.

\section{(c) (1)}

Este obra está licenciado com uma Licença Creative Commons Atribuição 4.0 Internacional.

${ }^{1}$ Enfermeira. Doutoranda em Enfermagem. Universidade Federal de São Paulo. São Paulo, SP, Brasil. (Q) ${ }^{2}$ Discente de Enfermagem. Universidade Federal de São Paulo. São Paulo, SP, Brasil. ()

${ }^{3}$ Enfermeira. Mestre em Ciências da Saúde. Universidade Federal de São Paulo. São Paulo, SP, Brasil.

${ }^{4}$ Psicóloga. Doutora em Ciências da Saúde. Psicóloga da Escola Paulista de Medicina. São Paulo, SP, Brasil.

${ }^{5}$ Enfermeira. Doutora em Enfermagem Materno-Infantil. Docente de Enfermagem da Universidade Federal de São Paulo. São Paulo, SP, Brasil.

${ }^{6}$ Enfermeira. Doutora em Enfermagem. Docente de Enfermagem da Universidade Federal de São Paulo. São Paulo, SP, Brasil. () 


\title{
ADHERENCE TO LIVER TRANSPLANTATION TREATMENT: AN INTEGRATIVE REVIEW
}

\begin{abstract}
Objective: to evaluate the interventions of the multidisciplinary team that provide improvements in adherence to treatment after liver transplantation.

Method: integrative review of the literature. The inclusion criteria were articles published between 2012 and 2017, in the Portuguese, English and Spanish languages, performed with the adult population that had undergone liver transplantation.

Results: the initial search resulted in 84 publications, with 10 studies being selected. The data were tabulated according to the relevance of the content, objectives, type of intervention, number of patients and level of evidence. Four analytical categories were found: educational interventions; adoption of an individual therapeutic plan; change in the immunosuppressive regimen; emotional and psychological support and strengthening of the support network.

Conclusion: the multidisciplinary team should provide information about the transplantation process, perform appropriate management of immunosuppression, regularly assess the patients for signs of depression and anxiety, investigate their beliefs and values, and institute an individualized care plan.

DESCRIPTORS: Liver transplant; Patient compliance; Adherence to medication; Patient care team; Review.
\end{abstract}

\section{ADHESIÓN AL TRATAMIENTO EN EL TRASPLANTE DE HÍGADO: REVISIÓN INTEGRATIVA}

\section{RESUMEN}

Objetivo: evaluar las intervenciones del equipo multiprofesional que promueven la mejoría de la adhesión al tratamiento tras el trasplante de hígado.

Método: revisión integrativa de la literatura. Se publicaron los criterios de inclusión entre 2012 y 2017, en los idiomas portugués, inglés y español, y estos se aplicaron en población adulta sometida al trasplante hepático.

Resultado: la búsqueda inicial resultó en 84 publicaciones, seleccionándose 10 estudios. Se tabularon los datos de acuerdo con la relevancia del contenido, objetivos, tipo de intervención, número de pacientes y nivel de evidencia. De eso, resultaron cuatro categorías analíticas: intervenciones educativas; adopción de un plan terapéutico individual; alteración del régimen inmunosupresor; soporte emocional, psicológico y fortalecimiento de la red de apoyo.

Conclusión: es necesario que el equipo multiprofesional provea informaciones acerca del proceso de trasplantación, realice el manejo adecuado de la inmunosupresión, evalúe regularmente el paciente cuanto a las señales de depresión, ansiedad, creencias y valores, e instituya un plan de cuidados individualizado.

DESCRIPTORES: Trasplante de hígado; Cooperación del paciente; Adhesión a la medicación; Equipo de asistencia al paciente; Revisión. 
O transplante de fígado possibilita ao paciente com doença hepática terminal a oportunidade de aumentar sua sobrevida, aliado à melhora da qualidade de vida. Entretanto, os resultados estão diretamente relacionados ao comprometimento regular do paciente com seu tratamento, incluindo a terapia imunossupressora e adequação do estilo de vida ${ }^{(1-3)}$.

A sobrevida do receptor de fígado pode variar entre 60 a $80 \%$ nos primeiros cinco anos após o procedimento, a depender do tipo de doença responsável pela indicação do transplante e de outras variáveis, como morbidades associadas, acesso a equipe de saúde e capacidade de desenvolver e gerenciar o autocuidado ${ }^{(3-4)}$.

O transplante envolve o risco inerente de rejeição do enxerto com consequente disfunção do órgão. Para minimizar este risco, os pacientes são submetidos a regimes imunossupressores e devem ser monitorados quanto aos sinais de falência do enxerto( ${ }^{(5)}$. Sendo assim, a não adesão ao tratamento influencia fortemente os desfechos clínicos nos transplantes, contribuindo inclusive para o aumento dos custos ao sistema de saúde ${ }^{(6-7)}$.

Uma meta-análise que incluiu 147 pesquisas publicadas entre 1981 e 2005, as quais abordavam a adesão à terapêutica imunossupressora após transplantes de órgãos sólidos, constatou que somente $20 \%$ desses estudos estavam relacionados aos receptores de fígado. Ainda assim, evidenciou que uma a cada 10 mortes de pacientes submetidos ao transplante de fígado estava relacionada a não adesão aos medicamentos imunossupressores. As taxas de não adesão foram de $36 \%$ entre os receptores de rim; $14,5 \%$ nos de coração e $6,7 \%$ entre os receptores de fígado(8).

Diversos fatores podem influenciar a adesão ao regime terapêutico ${ }^{(9)}$. A Organização Mundial de Saúde (OMS) elencou cinco dimensões que interferem na adesão, considerando a multidimensionalidade do processo: fatores relacionados ao paciente, ao tratamento, socioeconômicos, relacionados aos sistemas de saúde, e a doença ${ }^{(10)}$.

Os pacientes apresentam barreiras diferentes para a adesão ao tratamento, por isso é importante implementar intervenções adaptadas aos seus fatores de risco(11). Em 2009, uma revisão sistemática avaliou 12 estudos de intervenção que foram conduzidos para melhorar as taxas de adesão em pacientes que realizaram transplante de órgãos. Três deles abordavam especificamente a adesão em pacientes após o transplante de fígado, sendo apenas um com receptores adultos ${ }^{(12)}$. Esses estudos intervieram no prestador de cuidados de saúde e no ambiente de saúde, mas mostraram melhoria limitada na taxa de adesão. Mostraram ainda que nenhuma intervenção isolada foi superior no sentido de melhorar a adesão ao tratamento(12).

Desde então, muitos refinamentos ocorreram para ajudar a manter os pacientes envolvidos no tratamento. Tecnologias inovadoras de saúde e o estabelecimento de políticas de gerenciamento do paciente com doenças crônicas vêm demonstrando serem eficazes em aumentar a adesão do paciente ao tratamento ${ }^{(13-14)}$. Entretanto, há poucas evidências sobre as estratégias mais eficazes no manejo da adesão ao tratamento em populações específicas. Assim, torna-se de grande valia a investigação das intervenções que podem contribuir para a adesão no transplante de fígado, de maneira a fundamentar as ações da equipe multiprofissional junto a esses pacientes.

Frente ao exposto, o objetivo deste estudo foi mapear, por meio de uma revisão integrativa, as evidências existentes sobre intervenções propostas pela equipe multiprofissional, para evitar ou reduzir a falta de adesão ao tratamento farmacológico e não farmacológico em pacientes submetidos ao transplante de fígado. 
Revisão Integrativa (RI) da literatura, para identificação e análise dos estudos disponíveis nas bases de dados, que propicia a síntese de conhecimento produzido sobre um determinado tema, permite a visualização de lacunas de evidências na prática profissional e viabiliza a contextualização do pesquisador em determinada temática(15-16).

As etapas que constituíram a RI foram: identificação do tema e definição da pergunta norteadora; busca na literatura ou amostragem; extração dos dados dos estudos e compilação dos achados; avaliação dos estudos e interpretação dos resultados; e finalmente, a síntese do conhecimento ou apresentação da revisão integrativa propriamente dita ${ }^{(16)}$.

A identificação do tema teve início com a definição das palavras-chaves utilizadas para pesquisa inicial: Transplante de Fígado, Adesão ao tratamento, e intervenções da equipe multiprofissional. Para a definição e elaboração da pergunta de pesquisa, foi utilizada a estratégia de pesquisa PICO (17), um acrônimo, com origem na língua inglesa, utilizado para selecionar a população (Population): "pacientes adultos ( $>18$ anos) submetidos a transplante hepático"; a intervenção (Intervention): "intervenções para melhoria da adesão ao tratamento após o transplante de fígado; comparação (Comparison): "nível de adesão ao tratamento antes e após adoção de estratégias que visam melhorar a adesão"; e desfecho (Outcomes): "adesão ao tratamento após o transplante". Em seguida, foi identificada a questão de pesquisa: Quais são as intervenções realizadas pela equipe multiprofissional que contribuem para melhora da adesão ao tratamento no transplante de fígado?

A pesquisa foi realizada no mês de junho de 2017 em cinco bases de dados: Literatura Latino-americana e do Caribe em Ciências da Saúde (LILACS), Cumulative Index to Nursing and Allied Health Literature (CINAHL), Medical Literature Analysis and Retrieval System Online (Medline/Pubmed), Scientific Electronic Library Online (SciELO) e Biblioteca Virtual em Saúde (BVS), por meio dos descritores controlados "Adesão à Medicação/ Medication Adherence", "Transplante de Fígado/Liver Transplantation" e "Cooperação do Paciente/Patient Compliance", em diferentes combinações nos idiomas português e inglês, selecionados do Medical Subject Headings (MeSH) e Descritores em Ciências da Saúde (DeCS).

Os critérios de inclusão foram artigos com texto completo publicados entre 2012 e 2017, nos idiomas inglês, português e espanhol, realizados com pacientes adultos (idade maior ou igual a 18 anos) submetidos a transplante de fígado, independentemente do tipo de doador (doador vivo ou falecido) e da etiologia da insuficiência hepática. Qualquer intervenção aplicada para manter os pacientes seguindo o tratamento prescrito, farmacológico ou não farmacológico, foi considerada. A Tabela 1 apresenta a busca.

Tabela 1 - Detalhes da busca eletrônica. São Paulo, SP, Brasil, 2018.

\section{Linha Sequência da pesquisa}

\begin{tabular}{cl}
\hline 1 & (adherence in liver transplant) \\
\hline 2 & (interventions to improve adherence in liver transplant recipients) \\
\hline 3 & (Medication Adherence) AND (liver transplant or transplantation) \\
\hline 4 & (Patient Compliance) AND (adherence) AND (liver transplant or transplantation) \\
\hline 5 & (databases LILACS or Pubmed or CINAHL or BVS or SciELO) \\
\hline 6 & $\begin{array}{l}\text { (systematic or narrative or integrative or ethical or theorical or literature or comprehensive or } \\
\text { review or overview) }\end{array}$ \\
\hline 7 & Date: 2012-2017 \\
\hline 8 & Language: English, Spanish and Portuguese.
\end{tabular}


Como critério de exclusão considerou-se artigos sobre adesão abordando outras patologias e terapêuticas não relacionadas ao transplante de fígado.

Não foram impostas restrições quanto ao desenho dos estudos incluídos (controlado ou não controlado). Sendo assim, os estudos considerados foram ensaios clínicos controlados randomizados (ECCR) e, na ausência, ensaios clínicos não randomizados, estudos de coorte, caso controle ou estudos descritivos. Estudos mistos, realizados com outros tipos de transplante, desde que especificassem os receptores de fígado, também foram selecionados. As pesquisas secundárias (resumo, cartas ao editor, revisões e capítulos de livros) não foram incluídas. Guias de recomendações e anais de congressos foram excluídos, para evitar o potencial risco de viés devido a relatórios seletivos.

As estratégias adotadas para cada base descrita foram organizadas em pastas com o auxílio do programa Endnote $X 6 \AA$, que possibilita agrupamento de referências repetidas.

Depois de remover todas as duplicatas, foram selecionados os títulos e resumos de estudo e descartados aqueles que não se encaixaram nos critérios de inclusão. Estudos de intervenção que não abordaram a adesão ao tratamento como um resultado foram considerados inelegíveis. A extração dos dados dos realizada por meio de um instrumento validado, ${ }^{(17)}$ sendo realizada em duas fases: a) avaliação dos títulos e dos resumos de todos os estudos selecionados, de forma independente, por dois avaliadores; b) após a seleção dos resumos, mediante consenso dos avaliadores, seguiu-se para a avaliação do texto completo. A análise crítica dos artigos constituiu na leitura do estudo na íntegra, feita por dois pesquisadores de forma independente. A seguir, as publicações selecionadas foram recuperadas na íntegra para que pudessem ter a qualidade metodológica avaliada.

Os dois revisores avaliaram o nível de evidência dos estudos de forma independente, de acordo com a classificação da Oxford Centre for Evidence-Based Medicine, a qual estratifica os estudos em 1Ả (Revisão Sistemática, metanálise de ECCR), 1B (ECCR com intervalo de confiança de 95\%); 2A (Revisão Sistemática de Estudos de Coorte), 2B (Estudos de Coorte (incluindo Ensaio Clínico Randomizado de menor qualidade), 2C (Outcomes Research: observação de resultados terapêuticos e Estudos Ecológicos); 3A (Revisão Sistemática de Estudos de Caso-Controle), 3B (Estudo Individual de Caso-Controle); 4 (Série de Casos, estudos de Coorte e Caso-Controle de menor qualidade); e 5 (Opinião de Especialistas)(18).

Finalmente, as informações obtidas foram então sintetizadas e os resultados foram interpretados mediante análise descritiva, sendo construídas as categorias analíticas de acordo com os objetivos da revisão e pergunta norteadora.

\section{RESULTADOS}

Foram identificados 84 artigos nas bases de dados pesquisadas, sendo excluídos 32 por estarem em duplicidade. Dessa forma, 52 estudos foram elegíveis para a primeira seleção, realizada por meio da leitura dos títulos e resumos, etapa na qual se excluíram outros 38 por não atenderem aos critérios de inclusão. 14 artigos foram avaliados integralmente, dos quais quatro foram excluídos. Portanto, 10 artigos compuseram a amostra (Figura 1). 


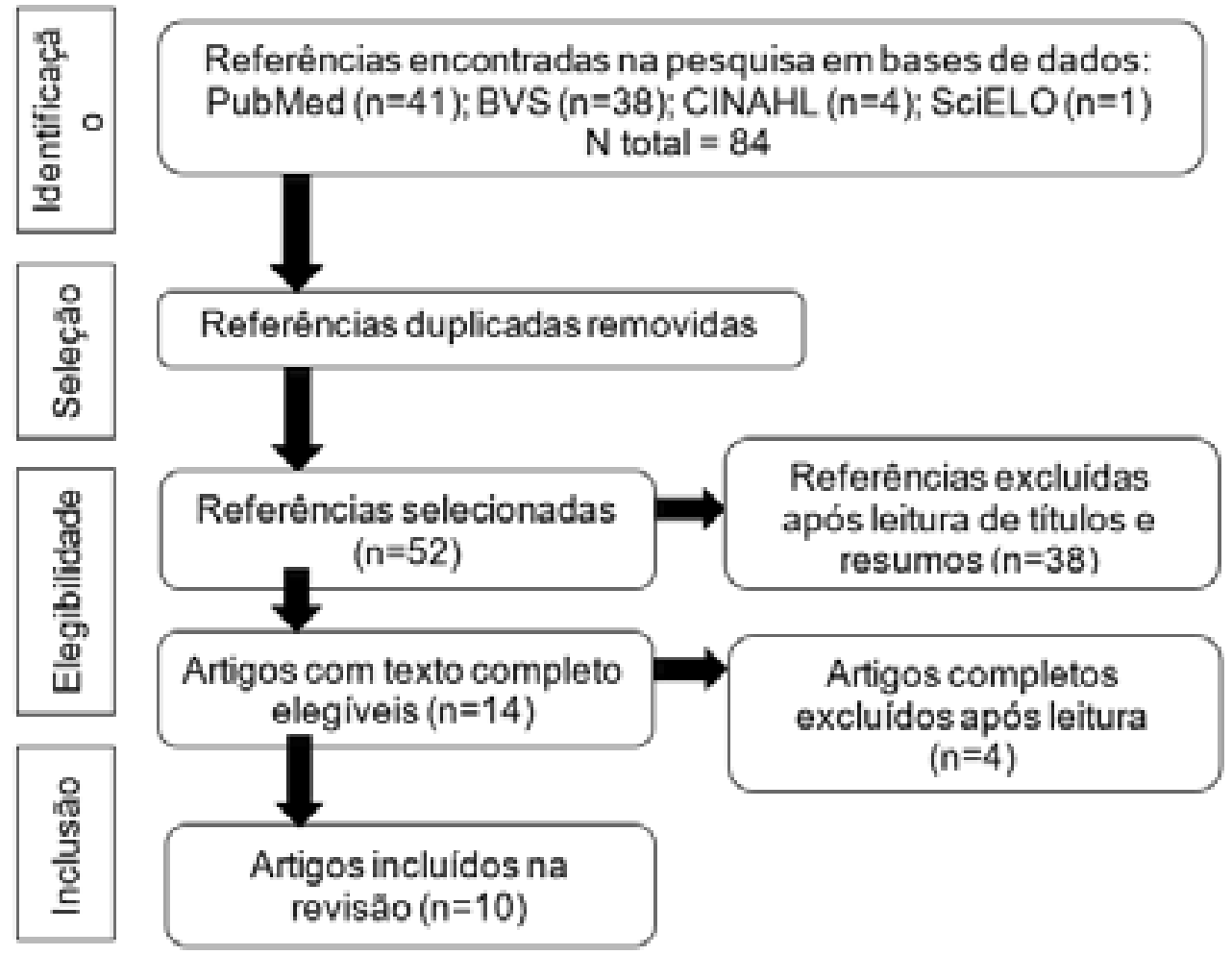

Figura 1 - Diagrama de inclusão e exclusão dos artigos encontrados nas bases de dados pesquisadas. São Paulo, SP, Brasil, 2018

Em seguida, para extração dos dados, realizou-se uma segunda leitura na íntegra dos 10 artigos selecionados, que foram organizados em tabelas, considerando os seguintes tópicos: título e ano da publicação, autores e nome do periódico; objetivo, tipo de estudo/ método, resultados e nível de evidência. O Quadro 1 apresenta os estudos segundo título, ano de publicação, autores e periódico.

Quadro 1 - Distribuição dos estudos segundo título, ano de publicação, autores e periódico. São Paulo, SP, Brasil, 2018 (continua)

\begin{tabular}{|l|c|l|c|}
\hline Título & Ano & Autores & Periódico \\
\hline $\begin{array}{l}\text { 1. Immunosuppressive Medication } \\
\begin{array}{l}\text { Adherence in Liver Transplant } \\
\text { Recipients }\end{array}\end{array}$ & 2016 (19) & $\begin{array}{l}\text { Promraja R, Dumronggittiguleb } \\
\text { W. Sirivatanauksornb Y, } \\
\text { Ruenroma A, Tovikkaib C, } \\
\text { Limsrichamrernb S, et al. }\end{array}$ & $\begin{array}{c}\text { Transplantation } \\
\text { Proceedings }\end{array}$ \\
\hline $\begin{array}{l}\text { 2. Systematic Pharmaceutical Educational } \\
\text { Approach to Enhance Drug Adherence in } \\
\text { Liver Transplant Recipients }\end{array}$ & 2016 & $\begin{array}{l}\text { Asavakarna S, } \\
\text { SirivatanauksornaY, Promrajb } \\
\text { R, Ruenrom A, Limsrichamrerna } \\
\text { S, Kositamongkola P, et al. }\end{array}$ & $\begin{array}{c}\text { Transplantation } \\
\text { Proceedings }\end{array}$ \\
\hline $\begin{array}{l}\text { 3. Adherence and treatment satisfaction in } \\
\text { liver transplant recipients }\end{array}$ & 2016 & $\begin{array}{l}\text { Albekairy AM, Alkatheri AM } \\
\text { Jarab A, Khalidi N, Althiab K, } \\
\text { Alshaya A, et al. }\end{array}$ & $\begin{array}{l}\text { The Saudi Journal } \\
\text { of } \\
\text { Gastroenterology }\end{array}$ \\
\hline $\begin{array}{l}\text { 4. Conversion of twice-daily to once-daily } \\
\text { tacrolimus is safe in stable adult living } \\
\text { donor liver transplant recipients }\end{array}$ & 2015 & $\begin{array}{l}\text { Kim SH, Lee SD, Kim YK, d Park } \\
\text { SJ. }\end{array}$ & $\begin{array}{c}\text { Hepatobiliary \& } \\
\text { Pancreatic Diseases } \\
\text { International }\end{array}$ \\
\hline
\end{tabular}




\begin{tabular}{|l|c|l|c|}
\hline $\begin{array}{l}\text { 5. Influence of intention to adhere, } \\
\text { beliefs and satisfaction about medicines } \\
\text { on adherence in solid organ transplant } \\
\text { recipients }\end{array}$ & 2014 (23) & $\begin{array}{l}\text { Hugon A, Roustit M, Lehmann } \\
\text { A, Saint-Raymond C, Borrel E, } \\
\text { Hilleret MN, et al. }\end{array}$ & Transplantation \\
\hline $\begin{array}{l}\text { 6. An observational study evaluating } \\
\text { tacrolimus dose, exposure, and } \\
\text { medication adherence after conversion } \\
\text { from twice- to once-daily tacrolimus in } \\
\text { liver and kidney transplant recipients(24) }\end{array}$ & 2014 & Bäckman L, Persson CA. & Ann Transplant \\
\hline $\begin{array}{l}\text { 7. Increased Medication Compliance of } \\
\text { Liver Transplant Patients Switched From } \\
\text { a Twice-Daily to a Once-Daily Tacrolimus- } \\
\text { Based Immunosuppressive Regimen'(25) }\end{array}$ & 2013 & Eberlin M, Otto G, Krämer I. & $\begin{array}{c}\text { Transplantation } \\
\text { Proceedings }\end{array}$ \\
\hline $\begin{array}{l}\text { 8. Rurality and other factors associated } \\
\text { with adherence to immunosuppressant } \\
\text { medications in community-dwelling solid- } \\
\text { organ transplant recipients }\end{array}$ & 2012 & $\begin{array}{l}\text { Sankaranarayanan J, Collier D, } \\
\text { Furasek A, Reardon T, Smith } \\
\text { LM, McCartan M, et al. }\end{array}$ & $\begin{array}{l}\text { Research in Social } \\
\text { and Administrative } \\
\text { Pharmacy }\end{array}$ \\
\hline $\begin{array}{l}\text { 9. Psychosocial predictors of adherence } \\
\text { after liver transplant in a single transplant } \\
\text { center in Portugal(27) }\end{array}$ & 2012 & $\begin{array}{l}\text { Diogo Telles-Correia, António } \\
\text { Barbosa, Inês Mega, Estela } \\
\text { Monteiro }\end{array}$ & $\begin{array}{c}\text { Progress in } \\
\text { Transplantation }\end{array}$ \\
\hline $\begin{array}{l}\text { 10. Immunosuppress and treatment } \\
\text { adherence, barriers to adherence and } \\
\text { quality of life in renal and liver transplant } \\
\text { recipients in Spain(28) }\end{array}$ & 2012 & Morales JM, Varo E, Lázaro P. & $\begin{array}{c}\text { Clinical } \\
\text { Transplantation }\end{array}$ \\
\hline
\end{tabular}

Os artigos foram avaliados quanto ao tipo de estudo, principais objetivos e tipo de intervenção estudada, para promover a adesão ao tratamento e nível de evidência. Foram encontrados nove (90\%) estudos transversais; um estudo prospectivo e multicêntrico (10\%). Como relação à amostra, esta variou entre 50 a 1.479 pacientes transplantados avaliados.

Visando facilitar a compreensão dos resultados obtidos, as investigações incluídas na revisão foram agrupadas em categorias analíticas, conforme descrito no Quadro 2.

Quadro 2 - Distribuição dos estudos segundo objetivo, tipo de estudo, tamanho da amostra, resultados e nível de evidência. São Paulo, SP, Brasil, 2018 (continua)

\begin{tabular}{|l|l|l|l|l|c|l|}
\hline $\mathbf{N}^{\circ}$ & Objetivo & $\begin{array}{l}\text { Tipo de } \\
\text { estudo }\end{array}$ & Amostra & Resultados & $\begin{array}{l}\text { Nível de } \\
\text { evidência }\end{array}$ & Categoria \\
\hline 1 & $\begin{array}{l}\text { Avaliar a adesão } \\
\text { à medicação } \\
\text { imunossupressora em } \\
\text { pacientes transplantados } \\
\text { hepáticos }\end{array}$ & $\begin{array}{l}\text { Trans- } \\
\text { versal, } \\
\text { em um } \\
\text { único } \\
\text { centro }\end{array}$ & $\begin{array}{l}50 \\
\text { pacientes }\end{array}$ & $\begin{array}{l}\text { Educação e } \\
\text { explicação da } \\
\text { importância dos } \\
\text { imunossupressores } \\
\text { ao paciente e à } \\
\text { família/cuidadores } \\
\text { são essenciais } \\
\text { para melhoria da } \\
\text { adesão. }\end{array}$ & 2C & $\begin{array}{l}\text { Intervenções } \\
\text { educativas: presença } \\
\text { de não adesão } \\
\text { relacionada ao déficit } \\
\text { de informação sobre o } \\
\text { tratamento. }\end{array}$ \\
\hline
\end{tabular}


Quadro 2 - Distribuição dos estudos segundo objetivo, tipo de estudo, tamanho da amostra, resultados e nível de evidência. São Paulo, SP, Brasil, 2018 (continução)

\begin{tabular}{|c|c|c|c|c|c|c|}
\hline 2 & $\begin{array}{l}\text { Implementar uma } \\
\text { abordagem educacional } \\
\text { farmacêutica para a } \\
\text { melhoria da adesão } \\
\text { à terapia de drogas } \\
\text { imunossupressoras no } \\
\text { hospital Siriraj(20). }\end{array}$ & $\begin{array}{l}\text { Trans- } \\
\text { versal, } \\
\text { em um } \\
\text { único } \\
\text { centro }\end{array}$ & $\begin{array}{l}50 \\
\text { pacientes }\end{array}$ & $\begin{array}{l}\text { Proporcionar } \\
\text { conhecimento } \\
\text { e atenção em } \\
\text { relação aos } \\
\text { imunossupressores } \\
\text { e o seu uso } \\
\text { melhoram a } \\
\text { adesão. } \\
\end{array}$ & $2 C$ & $\begin{array}{l}\text { Intervenções } \\
\text { educativas: presença } \\
\text { de não adesão } \\
\text { relacionada ao déficit } \\
\text { de informação sobre o } \\
\text { tratamento. }\end{array}$ \\
\hline 3 & $\begin{array}{l}\text { Identificar os fatores } \\
\text { que melhores predizem } \\
\text { a adesão à medicação } \\
\text { e explorar a relação } \\
\text { entre a satisfação com o } \\
\text { tratamento e a adesão à } \\
\text { medicação em paciente } \\
\text { submetidos ao transplante } \\
\text { hepático (21). }\end{array}$ & $\begin{array}{l}\text { Trans- } \\
\text { versal, } \\
\text { em um } \\
\text { único } \\
\text { centro }\end{array}$ & $\begin{array}{l}154 \\
\text { pacientes }\end{array}$ & $\begin{array}{l}\text { Pacientes mais } \\
\text { satisfeitos com } \\
\text { o tratamento } \\
\text { possuem melhor } \\
\text { adesão à terapia, } \\
\text { recomenda um } \\
\text { plano de cuidado } \\
\text { individual que } \\
\text { ajude na melhoria } \\
\text { da satisfação do } \\
\text { paciente com } \\
\text { tratamento e } \\
\text { consequentemente } \\
\text { a adesão. } \\
\end{array}$ & $2 C$ & $\begin{array}{l}\text { Adoção de um plano } \\
\text { terapêutico individual: } \\
\text { presença de não } \\
\text { adesão relacionada } \\
\text { ao não atendimento } \\
\text { das necessidades } \\
\text { específicas do } \\
\text { paciente. }\end{array}$ \\
\hline 4 & $\begin{array}{l}\text { Avaliar a segurança, } \\
\text { eficácia e adesão à } \\
\text { imunossupressão através } \\
\text { da conversão da dose de } \\
\text { Tacrolimus de duas vezes } \\
\text { ao dia para uma vez ao dia, } \\
\text { em adultos transplantados } \\
\text { hepáticos estáveis (por } \\
\text { doador vivo)(22). }\end{array}$ & $\begin{array}{l}\text { Trans- } \\
\text { versal, } \\
\text { em um } \\
\text { único } \\
\text { centro }\end{array}$ & $\begin{array}{l}229 \\
\text { pacientes }\end{array}$ & $\begin{array}{l}\text { Mudança para } \\
\text { dose (única). }\end{array}$ & $2 C$ & $\begin{array}{l}\text { Alteração do regime } \\
\text { imunossupressor: } \\
\text { presença de não } \\
\text { adesão relacionada } \\
\text { ao número de } \\
\text { doses diárias dos } \\
\text { medicamentos } \\
\text { imunossupressores. }\end{array}$ \\
\hline 5 & $\begin{array}{l}\text { Testar se os parâmetros de } \\
\text { um modelo adaptado da } \\
\text { teoria de comportamento } \\
\text { planejado, e mais } \\
\text { especificamente atitudes } \\
\text { que são influenciadas pelas } \\
\text { crenças e satisfação com a } \\
\text { medicação, podem predizer } \\
\text { a adesão em pacientes } \\
\text { transplantados de órgãos } \\
\text { sólidos }{ }^{(23)} \text {. }\end{array}$ & $\begin{array}{l}\text { Trans- } \\
\text { versal, } \\
\text { em um } \\
\text { único } \\
\text { centro }\end{array}$ & $\begin{array}{l}153 \\
\text { pacientes }\end{array}$ & $\begin{array}{l}\text { Avaliação } \\
\text { emocional. O } \\
\text { profissional } \\
\text { deve identificar } \\
\text { crenças negativas } \\
\text { em relação à } \\
\text { imunossupressão, } \\
\text { já que é um fator } \\
\text { que diminui a } \\
\text { adesão. }\end{array}$ & $2 C$ & $\begin{array}{l}\text { Suporte emocional, } \\
\text { psicológico e } \\
\text { apoio: presença } \\
\text { de não adesão } \\
\text { relacionada aos } \\
\text { fatores psicológicos, } \\
\text { emocionais, crenças e } \\
\text { valores. }\end{array}$ \\
\hline 6 & $\begin{array}{l}\text { O objetivo primário } \\
\text { é investigar o efeito } \\
\text { da conversão de dose } \\
\text { Tracrolimus de duas vezes } \\
\text { ao dia para uma vez ao } \\
\text { dia, em } 90 \text { dias após a } \\
\text { conversão em pacientes } \\
\text { transplantados renais e } \\
\text { hepáticos estáveis em } \\
\text { prática clínica rotineira. Um } \\
\text { segundo objetivo é avaliar } \\
\text { as mudanças na adesão à } \\
\text { medicação após a mudança } \\
\text { de dose de Tacrolimus }\end{array}$ & $\begin{array}{l}\text { Pros- } \\
\text { pectivo, } \\
\text { obser- } \\
\text { vacional } \\
\text { multicên- } \\
\text { trico }\end{array}$ & $\begin{array}{l}224 \\
\text { pacientes }\end{array}$ & $\begin{array}{l}\text { Mudança para } \\
\text { dose (única). }\end{array}$ & $2 \mathrm{~B}$ & $\begin{array}{l}\text { Alteração do regime } \\
\text { imunossupressor: } \\
\text { presença de não } \\
\text { adesão relacionada } \\
\text { ao número de } \\
\text { doses diárias dos } \\
\text { medicamentos } \\
\text { imunossu-pressores. }\end{array}$ \\
\hline
\end{tabular}


Quadro 2 - Distribuição dos estudos segundo objetivo, tipo de estudo, tamanho da amostra, resultados e nível de evidência. São Paulo, SP, Brasil, 2018 (continução)

\begin{tabular}{|c|c|c|c|c|c|c|}
\hline 7 & $\begin{array}{l}\text { Comparar as taxas de } \\
\text { adesão à medicação entre } \\
\text { pacientes transplantados } \\
\text { hepáticos ao longo do } \\
\text { tempo após o transplante } \\
\text { com o regime terapêutico } \\
\text { de Tacrolimus } \\
\text { (25). }\end{array}$ & $\begin{array}{l}\text { Trans- } \\
\text { versal, } \\
\text { em um } \\
\text { único } \\
\text { centro }\end{array}$ & $\begin{array}{l}63 \\
\text { pacientes }\end{array}$ & $\begin{array}{l}\text { Mudança de dose } \\
\text { (única) gerou } \\
\text { aumento da } \\
\text { adesão. }\end{array}$ & $2 C$ & $\begin{array}{l}\text { Alteração do regime } \\
\text { imunossupressor: } \\
\text { presença de não } \\
\text { adesão relacionada } \\
\text { ao número de } \\
\text { doses diárias dos } \\
\text { medicamentos } \\
\text { imunossupressores. }\end{array}$ \\
\hline 8 & $\begin{array}{l}\text { Examinar os fatores } \\
\text { associados ao instrumento } \\
\text { "Immunosuppressant } \\
\text { Therapy Adherence Scale" } \\
\text { (Escala de Adesão à Terapia } \\
\text { Imunossupressora) })^{(26)} \text {. }\end{array}$ & $\begin{array}{l}\text { Trans- } \\
\text { versal, } \\
\text { em um } \\
\text { único } \\
\text { centro }\end{array}$ & $\begin{array}{l}556 \\
\text { pacientes }\end{array}$ & $\begin{array}{l}\text { Intervenções } \\
\text { centradas no } \\
\text { paciente, com uso } \\
\text { de tecnologia para } \\
\text { subsidiar a tomada } \\
\text { de decisão. }\end{array}$ & $2 C$ & $\begin{array}{l}\text { Adoção de um plano } \\
\text { terapêutico individual: } \\
\text { presença de não } \\
\text { adesão relacionada } \\
\text { ao não atendimento } \\
\text { das necessidades } \\
\text { específicas. }\end{array}$ \\
\hline 9 & $\begin{array}{l}\text { Investigar quais fatores } \\
\text { psicossociais e psiquiátricos } \\
\text { determinam a adesão após } \\
\text { o transplante de fígado( }{ }^{(27)} \text {. }\end{array}$ & $\begin{array}{l}\text { Trans- } \\
\text { versal, } \\
\text { em um } \\
\text { único } \\
\text { centro }\end{array}$ & $\begin{array}{l}150 \\
\text { pacientes }\end{array}$ & $\begin{array}{l}\text { Plano terapêutico } \\
\text { de cuidados } \\
\text { individualizados } \\
\text { no pré-transplante } \\
\text { melhora a adesão } \\
\text { no pós-transplante. }\end{array}$ & $2 C$ & $\begin{array}{l}\text { Adoção de um plano } \\
\text { terapêutico individual: } \\
\text { presença de não } \\
\text { adesão relacionada } \\
\text { ao não atendimento } \\
\text { das necessidades } \\
\text { específicas. }\end{array}$ \\
\hline 10 & $\begin{array}{l}\text { Avaliar adesão } \\
\text { ao tratamento } \\
\text { imunossupressor e } \\
\text { identificar as barreiras } \\
\text { percebidas que afetam } \\
\text { a adesão, e também a } \\
\text { qualidade de vida de } \\
\text { pacientes submetidos } \\
\text { a transplante de rim e } \\
\text { fígado(28). }\end{array}$ & $\begin{array}{l}\text { Trans- } \\
\text { versal, } \\
\text { em um } \\
\text { único } \\
\text { centro }\end{array}$ & $\begin{array}{l}1479 \\
\text { pacientes }\end{array}$ & $\begin{array}{l}\text { O ajuste da } \\
\text { intensidade do } \\
\text { tratamento através } \\
\text { da redução da } \\
\text { frequência da } \\
\text { dose. }\end{array}$ & $2 C$ & $\begin{array}{l}\text { Alteração do regime } \\
\text { imunossupressor. }\end{array}$ \\
\hline
\end{tabular}

\section{DISCUSSÃO}

Os estudos que compuseram esta revisão, os quais tratam sobre a temática da não adesão ao tratamento entre pacientes submetidos ao transplante de fígado, foram produzidos predominantemente na América do Norte e Europa.

Com relação ao delineamento metodológico, a amostra foi composta majoritariamente por estudos do tipo coorte. Dos dez estudos, nove foram classificados com o nível de evidência 2C e apenas um como $2 \mathrm{~B}$.

Ressalta-se que as melhores evidências são obtidas em pesquisas com alta qualidade metodológica, pois são capazes de reunir informações que subsidiem a tomada de decisão clínica, tais como os ensaios clínicos randomizados. Entretanto, reconhece-se que os estudos de coorte são utilizados de maneira clássica para avaliar resultados de exposições a riscos ${ }^{(29)}$. Deste modo, frente à avaliação das intervenções adotadas para diminuir o risco de não adesão ao tratamento após o transplante de fígado, este poderia ser um dos delineamentos de pesquisa possíveis para investigação deste tipo de fenômeno.

Os dados encontrados nos estudos incluídos nesta revisão foram sintetizados em quatro categorias. A primeira delas, na qual foi incluída dois artigos, refere-se às 
"intervenções educativas - presença de não adesão relacionada ao déficit de informação sobre o tratamento".

As estratégias adotadas entre os estudos que compuseram essa categoria sugerem a otimização do processo de ensino e aprendizagem como uma ferramenta útil para aumento do nível de adesão ao tratamento. A inclusão de familiares no ensino do uso de medicamentos e o fornecimento de informações sobre o processo de transplantação, associado ao contato direto com profissional responsável pelo acompanhamento clínico do paciente, parece ter impacto também nos índices de adesão(19-20).

Cabe ressaltar que o comportamento de adesão não deve ser reduzido ao cumprimento das recomendações profissionais, mas ao resultado da exposição a situações de aprendizagem (medidas de educação em saúde) e de estratégias de enfrentamento desenvolvidas pelo paciente e família a partir dessas intervenções, que terão impacto na qualidade de vida, e especificamente no transplante de fígado, na sobrevida do enxerto e do paciente(4).

Os pacientes mantêm a condição de portadores de doenças crônicas após o transplante e, por essa razão, o uso de medicamentos faz parte de sua rotina, acarretando riscos de agravos à saúde relacionados a essa condição.

É fundamental que o paciente e sua família sejam capazes de compreender as circunstancias que envolvem o tratamento após o transplante de fígado; que aprendam a lidar com os medicamentos; mudem hábitos de higiene com o objetivo de prevenir processos infecciosos; adaptem-se a diversas alterações, como na autoimagem, além de questões sociais, como, por exemplo, o retorno às atividades laborais. Desta maneira, é essencial observar como o paciente se comporta e percebe o tratamento. Reconhecer as histórias, os hábitos e as habilidades dos pacientes os coloca como foco da intervenção e do cuidado em saúde ${ }^{(23,30)}$.

A categoria com o maior número de artigos foi "alteração do regime imunossupressor - presença de não adesão relacionada ao número de doses diárias dos medicamentos imunossupressores", que tratou das dificuldades de adesão ocasionadas pelo uso de diversos medicamentos concomitantemente.

A intervenção adotada nos quatro trabalhos incluídos nessa categoria foi à alteração da posologia, com a diminuição de doses (de duas doses para uma dose diária de imunossupressor). A partir da adoção dessa estratégia, evidenciou-se significativa redução dos índices de não adesão, estabilização dos níveis séricos das drogas e ausência de complicações sistêmicas - renais, cardiológicas e hepáticas ${ }^{(22-25,28)}$. Portanto, a simplificação dos esquemas terapêuticos é mais uma recomendação útil, contribuindo para alcance dos melhores resultados relacionados à adesão imunossupressora.

Outra categoria foi a relacionada à "adoção de um plano terapêutico individualpresença de não adesão relacionada ao não atendimento das necessidades específicas do paciente", composta por três artigos ${ }^{(21,26-27)}$.

Diante da complexidade do paciente submetido ao transplante, é fundamental a atuação de uma equipe, preparada para atender as demandas dos pacientes, em todos os níveis de atenção à saúde e de forma holística. Deve-se considerar a duração, a diversidade de cuidados e a natureza das recomendações realizadas pela equipe como fatores que intervêm na adesão ${ }^{(31)}$.

A última categoria foi composta por apenas um trabalho(23) e associa-se ao "suporte emocional, psicológico e apoio - presença de não adesão relacionado a fatores psicológicos, emocionais, crenças e valores.". Diversos fatores têm sido relacionados aos comportamentos de não adesão ao tratamento, desde diagnóstico de depressão e ansiedade, até inadequação ao novo estilo de vida e dificuldades de reinserção na vida familiar e cotidiana após o transplante ${ }^{(32)}$. 
Outras questões de ordem social ou associadas a características próprias do paciente, como ser do sexo masculino, com baixo suporte social(33), ou também relacionados ao sistema de saúde, como a falta ou não de oferecimento da medicação, dificuldade de acesso aos centros transplantadores ${ }^{(34)}$, podem ser relacionadas à não-adesão ao tratamento pós transplante.

Na maioria dos estudos em adesão a saúde, existe a preocupação com a discordância entre as orientações dos profissionais de saúde e os comportamentos dos pacientes. Diante deste cenário, há várias posturas, mecanismos e intervenções para modificar os comportamentos considerados de risco. A presente revisão evidenciou que, quando se trata de não adesão ao tratamento pós-transplante, a área mais estudada é a não adesão aos medicamentos imunossupressores.

Poucos estudos abordam medidas eficazes que promovem mudanças de comportamento relacionadas ao estilo de vida desejável após o transplante. Ressaltamos que ambas as intervenções têm relação estreita com os resultados a longo prazo após o transplante de fígado. Sendo assim, sugere-se o desenvolvimento de estudos que avaliem as intervenções da equipe multiprofissional que promovam a adesão ao tratamento pós transplante de forma global, não se atendo apenas aos aspectos inerentes à imunossupressão.

Foi identificado que os estudos apresentados fazem uma análise da adesão, baseadas na distribuição de adesão e não adesão na população transplantada, e trazem as intervenções e os mecanismos que devem ser seguidos para atingir os melhores resultados. Entretanto, não discutem os valores e as contingências relacionadas à manutenção de comportamento de risco relacionado a não adesão. Ou seja, o modo como os pacientes enfrentam as situações ameaçadoras, suas crenças e hábitos são variáveis que afetam as respostas dos indivíduos, e esse comportamento deve ser levado em consideração quando avaliamos a não adesão ao transplante de fígado.

Talvez isto esteja relacionado à própria definição clássica de adesão ao tratamento, que não considera a mudanças no repertório comportamental e cognitivo dos pacientes, a partir das intervenções implementadas (deixar de fumar e beber, prevenir situação evocadoras de estresses, adotar hábitos sexuais seguros, adaptar a rotina para ter uma dieta mais saudável com incorporação da atividade física), apenas consideram se o paciente cumpre com as orientações de um profissional de saúde de forma objetiva (tomar medicamentos ou comparecer a rotina de consultas e exames) $)^{(35-36)}$.

Acredita-se que as questões abordadas nesta revisão integrativa podem auxiliar a tomada de decisão do enfermeiro e dos demais profissionais de saúde, no sentido de mapear e reconhecer os fatores de riscos relacionados a não adesão ao tratamento no transplante de fígado, sobretudo sugerindo estratégias e intervenções que estão associadas ao melhor monitoramento do paciente e o aumento da adesão ao tratamento ${ }^{(35,37)}$.

Faltam estudos multicêntricos que avaliem a adesão globalmente, incluindo a adesão às recomendações e orientações da equipe e não apenas aos aspectos relacionados à terapia imunossupressora. A maioria dos estudos discutem fatores de risco associados à não adesão e não versam sobre ações para a melhoria dos níveis da mesma. Além disso, são utilizados diferentes métodos para aferir a adesão, e isso faz com que seja difícil comparar os resultados isoladamente.

As limitações deste estudo residem no déficit de investigações controladas com maior rigor no delineamento metodológico, as quais permitem maiores comparações e extrapolamentos dos resultados obtidos por meio da RI. 
adultos, submetidos a transplante de fígado, apontados pelos estudos analisados, são de quatro naturezas: a) intervenções educativas - presença de não adesão relacionada ao déficit de informação sobre o tratamento; b) adoção de um plano terapêutico individual- presença de não adesão relacionada ao não atendimento das necessidades específicas do paciente; c) alteração do regime imunossupressor - presença de não adesão relacionada ao número de doses diárias dos medicamentos imunossupressores; d) suporte emocional, psicológico e apoio - presença de não adesão relacionado a fatores psicológicos, emocionais, crenças e valores.

Sendo assim, a equipe multiprofissional deve prover informações sobre o processo de transplantação ao paciente e sua família, realizar o manejo adequado da imunossupressão com foco na simplificação do esquema terapêutico, avaliar regularmente o paciente quanto aos sinais de depressão, ansiedade, crenças e valores, e instituir um plano de cuidados individualizado.

Neste sentido, a incorporação de modelos de atenção à saúde que deem conta de tratar da temática "adesão ao tratamento nos pós-transplante" devem ser abrangentes, multidisciplinares e com foco nas necessidades individuais de cada paciente, incorporando a perspectiva analítico comportamental à avaliação, de forma que seja possível levar em consideração o indivíduo e suas relações com o mundo que o cerca.

\section{AGRADECIMENTOS}

Agradecemos ao Conselho Nacional de Desenvolvimento Científico (CNPq) que financiou o projeto de pesquisa que deu origem a esse artigo, que compõe o Edital Universal CNPq intitulado "Impacto do atendimento multiprofissional na adesão ao transplante de fígado". A autora principal também agradece à Agência de Fomento à Pesquisa pelo financiamento do Programa de Bolsas Demanda Social Conselho Nacional de Desenvolvimento Científico (CNPq).

\section{REFERÊNCIAS}

1. Martin P, Martini A, Feng S, Brown Jr RS, Fallon M. Evaluation for Liver Transplantation in Adults: 2013 Practice Guideline by the American Association for the Study of Liver Diseases and the American Society of Transplantation. Hepatology [Internet]. 2014 [acesso 01 set 2017]; 59(3). Disponível em: https://www. aasld.org/sites/default/files/guideline documents/Evaluation for\%20LT in Adults hep26972 0.pdf.

2. Ming-Ming X, Brown RS Jr. Liver Transplantation for the Referring Physician. Clin Liver Dis. [Internet]. 2015 [acesso 23 set 2017]; 19(1). Disponível em: https://doi.org/10.1016/j.cld.2014.09.008.

3. Drent G, Moons P, Geest S, Kleibeuker JH, Haagsma EB. Symptom experience associated with immunosuppressive drugs after liver transplantation in adults: possible relationship with medication noncompliance? Clin Transplant. [Internet]. 2008 [acesso 22 ago 2017]; 22(6). Disponível em: http://dx.doi. org/10.1111/j.1399-0012.2008.00864.x.

4. Mendes KDS, Rossin FM, Ziviani LC, Castro-e-Silva O, Galvão CM. Necessidades de informação de candidatos ao transplante de fígado: o primeiro passo do processo ensino-aprendizagem. Rev. Gaúcha Enferm. [Internet]. 2012 [acesso 28 ago 2017]; 33(4). Disponível em: http://dx.doi.org/10.1590/S198314472012000400012.

5. Morrissey PE, Flynn ML, Lin S. Medication noncompliance and its implications in transplant recipients. Drugs [Internet]. 2007 [acesso 17 ago 2017]; 67(10). Disponível em: https://doi.org/10.2165/00003495$200767100-00007$.

6. Pinsky BW, Takemoto SK, Lentine KL, Burroughs TE, Schnitzler MA, Salvalaggio PR. Transplant Outcomes and Economic Costs Associated with Patient Noncompliance to Immunosuppression. Am J Transplan. [Internet]. 2009 [acesso 18 ago 2017]; 11(9). Disponível em: https://doi.org/10.1111/j.1600- 
7. Chisholm MA, Mulloy LL, DiPiro JT. Comparing renal transplant patients' adherence to free cyclosporine and free tacrolimus immunosuppressant therapy. Clin Transplant. [Internet]. 2005 [acesso 19 ago 2017]; 19(1). Disponível em: https://doi.org/10.1111/j.1399-0012.2004.00301.x.

8. Dew MA, Martini AF, VitoDabbs A, Myaskovsky L, Steel J, Unruh M, et al. Rates and Rates and Risk Factors for Nonadherence to the Medical Regimen After Adult Solid Organ Transplantation. Transplantation. [Internet]. 2007 [acesso 19 ago 2017]; 83(7). Disponível em: https://doi.org/10.1097/01. tp.0000258599.65257.ab.

9. Johnson MJ. The Medication Adherence Model: A Guide for Assessing Medication Taking. Research Theory Nurs Pract. [Internet]. 2002 [acesso 17 ago 2017]; 16(3). Disponível em: https://doi.org/10.1891/ rtnp.16.3.179.53008.

10. Sabate E. Adherence to Long-Term Therapies: evidence for action. Geneva: World Health Organization, 2003.

11. Low JK, Williams A, Manias E, Crawford K. Interventions to improve medication adherence in adult kidney transplant recipients: a systematic review. Nephrology Dialysis Transplantation. [Internet.] 2015 [acesso 19 Jun 2017]; 30(5). Disponível em: https://doi.org/10.1093/ndt/gfu204.

12. Bleser L, Matteson M, Dobbels F, Russell C, Geest S. Interventions to improve medication adherence after transplantation: a systematic review. Transplantation Internacional. [Internet]. 2009 [acesso 26 ago 2017]; 22(8). Disponível em: https://doi.org/10.1111/j.1432-2277.2009.00881.x.

13. Cutler DM, Everett W. Thinking Outside the Pillbox - Medication Adherence as a Priority for Health Care Reform. N Engl J Med. [Internet]. 2010 [acesso em 16 set 2017]; 362(17). Disponível em: http://www. nejm.org/doi/full/10.1056/NEJMp1002305\#t=article.

14. Patel S, Jacobus-Kantor L, Marshall L, Ritchie C, Kaplinski N, Khurana PS, et al. Mobilizing your medications: an automated medication reminder application for mobile phones and hypertension medication adherence in a high-risk urban population. J Diabetes ScienTechn. [Internet]. 2013 [acesso 28 ago 2017]; 7(3). Disponível em: https://doi.org/10.1177/193229681300700307.

15. Souza MT, Silva MD, Carvalho R. Revisão Integrativa: o que é e como fazer. Einstein. [Internet]. 2010 [acesso 15 ago 2017]; 8(1). Disponível em: http://dx.doi.org/10.1590/s1679-45082010rw1134.

16. Forrest JL, Kupiec LM. Evidence-based Decision Making: Introduction and Formulating Good Clinical Questions. [Internet]. 2014 [Acesso 17 jul 17]; 1(3). Disponível em: https://www.dentalcare.com/en-us/ professional-education/ce-courses/ce311.

17. Santos CMC, Pimenta CAM, Nobre MRC. The PICO strategy for the research question construction and evidence search. Rev. Latino-Am. Enfermagem. [Internet]. 2007 [acesso 2017 ago 17]; 15(3) Disponível em: http://dx.doi.org/10.1590/S0104-11692007000300023.

18. Centre for Evidence-Based Medicine. Oxford centre for evidence-based medicine - levels of evidence (March 2009). [Internet]. 2009 [acesso 16 jun 2017]. Disponível em: http://www.cebm.net/oxford-centreevidence-based-medicine-levels-evidence-march-2009/.

19. Promraj R, Dumronggittigule W, Sirivatanauksorn Y, Ruenrom A, Tovikkai C, Limsrichamrern S, et al. Immunosuppressive medication adherence in liver transplant recipients. Transplant. Proc. [Internet]. 2016 [acesso 13 jun 2017]; 48(4). Disponível em: https://doi.org/10.1016/j.transproceed.2015.12.097.

20. Asavakarna S, Sirivatanauksorna Y, Promrajb R, Ruenrom A, Limsrichamrerna $S$, Kositamongkola $P_{1}$ et al. Systematic pharmaceutical educational approach to enhance drug adherence in liver transplant recipients. Transplant. Proc. [Internet]. 2016 [acesso 13 jun 2017]; 48(4). Disponível em: https://doi. org/10.1016/j.transproceed.2015.12.100.

21. Albekairy AM, Alkatheri AM, Jarab A, Khalidi N, Althiab K, Alshaya A, et al. Adherence and treatment satisfaction in liver transplant recipients. Saudi J Gastroenterol. [Internet]. 2016 [acesso 20 jun 17]; 22(2). 
22. SH Kim, SD Lee, YK Kim, SJ Park. Conversion oft wice-daily to once-daily tacrolimus is safe in stable adult living donor liver transplant recipients. Hepatobiliary Pancreat Dis Int. [Internet]. 2015 [acesso 13 jun 2017]; 14(4). Disponível em: https://doi.org/10.1016/S1499-3872(15)60378-2.

23. Hugon A, Roustit M, Lehmann A, Saint-Raymond C, Borrel E, Hilleret MN, et al. Influence of intention to ad here, beliefs and satisfaction about medicines on adherence in solid organ transplant recipients.

Transplant. [Internet]. 2014 [acesso 13 jun 2017]; 98(2). Disponível em: https://doi.org/10.1097/ TP.0000000000000221.

24. Bäckman L, Persson CA. An observational study evaluating tacrolimus dose, exposure, and medication adherence after conversion from twice- to once-daily tacrolimus in liver and kidney transplant recipients. Ann Transplant. [Internet]. 2014 [acesso 13 jun 2017]; (19). Dispovível em: http://doi.org/10.12659/ AOT.890101.

25. Eberlin, M, Otto G, Krämer I. Increased Medication Compliance of Liver Transplant Patients Switched From a Twice-Daily to a Once-Daily Tacrolimus-Based Immunosuppressive Regimen. Transplant. Proc. [Internet]. 2013 [acesso 13 jun 2017]; 45(6). Disponível em: https://doi.org/10.1016/j. transproceed.2012.10.037.

26. Sankaranarayanan J, Collier D, Furasek A, Reardon T, Smith LM, McCartan M, et al. Rurality and other factors associated with adherence to immunosuppressant medications in community-dwelling solid-organ transplant recipients. Research Soc Admin Pharm. [Internet]. 2012 [acesso 13 jun 2017]; 8(3). Disponível em: https://doi.org/10.1016/j.sapharm.2011.04.001.

27. Telles-Correia D, Barbosa A, Mega I, Monteiro E. Psychosocial predictors of adherence after liver transplant in a single transplant center in Portugal. Progress transplant. [Internet]. 2012 [acesso 14 jun 2017];22(1). Disponível em: https://doi.org/10.7182/pit2012569.

28. Morales JM, Varo E, Lázaro P. Immunosuppressant treatment adherence, barriers to adherence and quality of life in renal and liver transplant recipients in Spain. ClinTransplant. [Internet]. 2012 [acesso 14 jun 2017]; 26(2). Disponível em: https://doi.org/10.1111/j.1399-0012.2011.01544.x.

29. Lazcano-Ponce E, Fernández E, Salazar-Martínez E, Hernández-Ávila M. Estudios de cohorte. Metodología, sesgos y aplicación. Salud Pública México. [Internet]. 2000 [acesso 17 jul 2017]; 42(3). Disponível em: http://doi.org/10.1590/S0036-36342000000300010.

30. Jaarsma T, Nikolova-Simons M, Van Der Wal MHL. Nurses' strategies to address self-care aspects related to medication adherence and symptom recognition in heart failure patients: An in-depth look. Heart Lung. [Internet]. 2012 [acesso 2 ago 2017]; 41(6). Disponível em: https://doi.org/10.1016/j. hrtlng.2012.03.003.

31. Berben L, Dobbels F, Engberg S, Hill MN, De Geest S. Anecological perspective on medication adherence. Western J Nursing Research. [Internet]. 2012 [acesso 29 ago 2017]; 34(5). Disponível em: https://doi.org/10.1177/0193945911434518.

32. Bautista LE, Vera-Cala LM, Colombo C, Smith P. Symptoms of depression and anxiety and adherence to antihypertensive medication. American J Hypertension. [Internet]. 2012 [acesso 16 set 2017]; 25(4).

Disponível em: https://doi.org/10.1038/ajh.2011.256.

33. Rodrigue JR, Nelson DR, Hanto DW, Reed Al, Curry MP. Patient-reported immunosuppression nonadherence 6 to 24 months after liver transplant: Association with Pretransplant Psychosocial Factors and Perceptions of Health Status Change. Progress Transplant. [Internet] 2013 [acesso 16 set 2017]; 23(4). Disponível em: http://doi.org/10.7182/pit2013501.

34. Bender BG. Can Health Care Organizations Improve Health Behavior and Treatment Adherence? Population Health Management. [Internet] 2014 [acesso 11 set 2017]; 17(2). Disponível em: https://doi. org/10.1089/pop.2013.0045.

35. Mendes KDS, Silva Junior OC, Ziviani LC, Rossin FM, Zago MMF, Galvão CM. Educational intervention 
for liver transplantation candidates. Rev. Latino Am. Enfermagem. [Internet]. 2013 [acesso 16 set 2017]; 21(1). Disponível em: http://dx.doi.org/10.1590/S0104-11692013000100018.

36. Moraes ABA, Rolim GS, Costa Jr AL. O processo de adesão numa perspectiva analítico comportamental. Rev Bras De Ter Comp. [Internet]. 2009 [acesso 10 jun 2017]; (02). Disponível em: http:// pepsic.bvsalud.org/pdf/rbtcc/v11n2/v11n2a09.pdf.

37. Abedini S, Gøransson L, Cockburn E, Kilany S, Holdaas H. Immunosuppression Adherence in Stable Kidney Transplant Patients Converted From Immediate- to Prolonged-Release Tacrolimus in Clinical Practice: A Norwegian Study. Transplant Direct. [Internet]. 2018 [acesso 1 mar 2018]; 4. Disponível em: https://www.researchgate.net/publication/322269209 Immunosuppression Adherence in Stable Kidney Transplant Patients Converted From Immediate- to Prolonged-Release Tacrolimus in Clinical Practice A Norwegian Study.

Recebido: 09/03/2018

Finalizado: 18/01/2019

Autor Correspondente:

Priscilla Caroliny de Oliveira

Universidade Federal de São Paulo

R. Napoleão de Barros 754 - 04024002 - São Paulo, SP, Brasil

E-mail: pri.transplante@gmail.com

\section{Contribuição dos autores:}

Contribuições substanciais para a concepção ou desenho do estudo; ou a aquisição, análise ou interpretação de dados do estudo - PCO, FPD, SM, JS, BAR

Elaboração e revisão crítica do conteúdo intelectual do estudo - PCO, HBP, JS, BAR

Aprovação da versão final do estudo a ser publicado - PCO, JS, BAR

Responsável por todos os aspectos do estudo, assegurando as questões de precisão ou integridade de qualquer parte do estudo - PCO 